\title{
The local Hölder exponent for the dimension of invariant subsets of the circle
}

\author{
CARLO CARMINATI $\dagger$ and GIULIO TIOZZO $\ddagger$ \\ $\dagger$ † Dipartimento di Matematica, Università di Pisa, Largo Pontecorvo 5, 56127 Pisa, Italy \\ (e-mail: carminat@dm.unipi.it) \\ $\ddagger$ Yale University, 10 Hillhouse Avenue, New Haven, CT 06511, USA \\ (e-mail: giulio.tiozzo@yale.edu)
}

(Received 2 September 2015 and accepted in revised form 9 November 2015)

\begin{abstract}
We consider for each $t$ the set $K(t)$ of points of the circle whose forward orbit for the doubling map does not intersect $(0, t)$, and look at the dimension function $\eta(t):=\mathrm{H} \cdot \operatorname{dim} K(t)$. We prove that at every bifurcation parameter $t$, the local Hölder exponent of the dimension function equals the value of the function $\eta(t)$ itself. A similar statement holds for general expanding maps of the circle: namely, we consider the topological entropy of the map restricted to the survival set, and obtain bounds on its local Hölder exponent in terms of the value of the function.
\end{abstract}

\section{Introduction}

The theory of open dynamical systems, also known as dynamical systems 'with holes', was developed to model physical phenomena with escape of mass. One of the simplest models that can be analyzed rigorously is the case of expanding maps of the circle $\mathbb{S}:=\mathbb{R} / \mathbb{Z}$, where the hole is an interval with a fixed point on its boundary.

More precisely, we shall fix an integer $d \geq 2$ once and for all, and consider $g(x):=d x$ mod 1 the map given by multiplication by $d$ on the circle $\mathbb{S}$. For each $t \in[0,1]$, let us define the set

$$
K(t):=\left\{x \in \mathbb{R} / \mathbb{Z}: g^{k}(x) \notin(0, t) \forall k \geq 0\right\}
$$

of elements whose forward orbit under $g$ does not intersect the interval $(0, t)$. For each $t$, the set $K(t)$ is compact and forward invariant for $g$. One can see, immediately, that $K(0)=\mathbb{S}$ and $K(1)=\{0\}$; moreover, $K(t)$ is a decreasing family of sets, in the sense that $s<t$ implies $K(s) \supseteq K(t)$.

We shall consider the dimension function

$$
\eta(t):=\mathrm{H} \cdot \operatorname{dim} K(t),
$$

which gives the Hausdorff dimension of the set $K(t)$ as a function of the parameter $t$. 


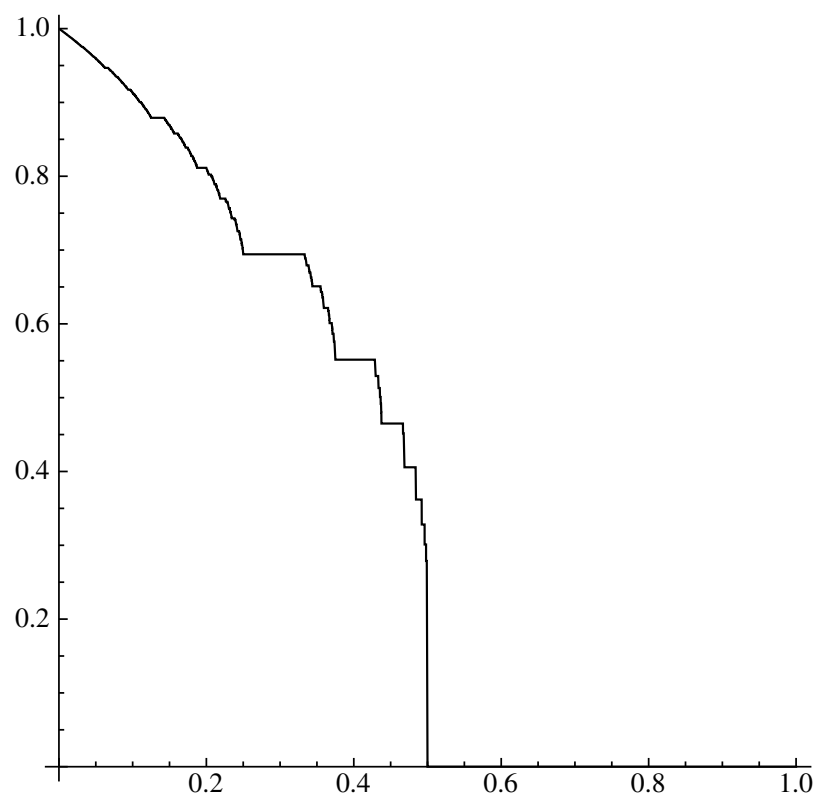

FIGURE 1. The dimension function $\eta(t)$ for $d=2$.

The function $\eta(t)$ was introduced by Urbański [14], who proved that it is continuous, but not globally analytic. In fact, he showed that the dimension function is a 'devil's staircase', that is, it is locally constant almost everywhere (see Figure 1).

In order to describe the finer analytical properties of $\eta(t)$, we shall call the set of parameters $t$ for which the set-valued function $t \mapsto K(t)$ is locally constant at $t$ the stable set, and the complement of the stable set will be called the bifurcation set and will be denoted by $\mathcal{U}$. Clearly, the dimension function is locally constant on the stable set (actually, $\eta$ is locally constant only on the stable set; see after Theorem 1).

We shall prove that, on the bifurcation set, the dimension function $\eta(t)$ has the following strong self-parametrizing property.

At every bifurcation parameter, the local Hölder exponent of the dimension function equals the value of the function itself.

To state the result precisely, let us define the local Hölder exponent of a function $f: I \rightarrow \mathbb{R}$ at a point $x \in I$ as the limit

$$
\alpha(f, x):=\liminf _{x^{\prime} \rightarrow x} \frac{\log \left|f(x)-f\left(x^{\prime}\right)\right|}{\log \left|x-x^{\prime}\right|} .
$$

The main theorem is the following.

THEOREM 1. Let $\geq 2$. Then, for each parameter $t$ in the bifurcation set, the local Hölder exponent of $\eta$ at $t$ equals $\eta(t)$ : that is,

$$
\alpha(\eta, t)=\eta(t) .
$$


As a corollary, the dimension function is always locally Hölder continuous except at $t=(d-1) / d$ (where $\eta(t)=0)$ and becomes more and more regular as $t$ tends to zero. Actually, $\eta$ is known to be differentiable at $t=0$ (see [7]). At $t=(d-1) / d$, the function is not Hölder continuous, and we shall show that its modulus of continuity is of order $(\log \log (1 / x)) / \log (1 / x)$ (see Proposition 15). Moreover, the intervals where the function is constant correspond precisely to the connected components of the stable set, and these are characterized in terms of Lyndon words (see below).

The dimension function $\eta$ is directly related to other quantities which have been widely studied. First, if we denote the set of points which do not fall into the hole under the first $n$ iterates by $M_{n}:=\left\{x \in \mathbb{S}: g^{k}(x) \notin(0, t)\right.$ for $\left.k=0, \ldots, n-1\right\}$, we can define the escape rate $\gamma$ to be

$$
\gamma:=\lim _{n \rightarrow \infty}-\frac{1}{n} \log \left|M_{n}\right|
$$

The escape rate is directly related to $\eta$ by the formula

$$
\eta=1-\frac{\gamma}{\log d} \text {. }
$$

In particular, the asymptotic behaviour of $\gamma$ in the 'small hole' case is quite well understood (see, for example, [7] and [3]); this gives an asymptotic expansion of $\eta(t)$ as $t \rightarrow 0$. Moreover, if we denote the topological entropy of the restriction of the map $g$ to $K(t)$ by $H(t)$, then (see, for example, [15])

$$
H(t)=\eta(t) \cdot \log d .
$$

Actually, Urbański [14] casts the problem in the following more general setting. Recall that a $C^{1}$ map $f: \mathbb{S} \rightarrow \mathbb{S}$ is called expanding if $\inf _{x \in \mathbb{S}}\left|f^{\prime}(x)\right|>1$. Let $f: \mathbb{S} \rightarrow \mathbb{S}$ be an expanding map of the circle of degree $d$, and let us assume that zero is a fixed point of $f$. He considers the survival set

$$
K_{f}(t):=\left\{x \in \mathbb{S}: f^{k}(x) \notin(0, t) \forall k \geq 0\right\}
$$

and the topological entropy of $f$ restricted to the survival set, that is, the function

$$
H(t):=h_{\mathrm{top}}\left(\left.f\right|_{K_{f}(t)}\right) .
$$

As is well known, in this case $f$ is topologically conjugate to the map $g(x):=d x \bmod 1$, and the conjugacy is Hölder continuous. Thus we have the following corollary.

COROLlary 2. Let $f$ be an expanding $C^{1}$ map of the circle, of degree $d$, and let $H(t)$ be defined as above. Then $H$ is locally Hölder continuous at points $t$ for which $H(t)>0$, and there are positive constants $C_{1}, C_{2}$, which depend only on $f$, for which the local Hölder exponent satisfies

$$
\alpha(H, t) \geq C_{1} H(t) \quad \text { for all } t \in[0,1]
$$

and, for every $t$ in the bifurcation set,

$$
\alpha(H, t) \leq C_{2} H(t) .
$$

As a consequence, $H$ is not locally Hölder continuous at the point

$$
t_{\star}:=\sup \{t: H(t)>0\} .
$$


In a recent paper [1], Bandtlow and Rugh independently obtain similar results using thermodynamic formalism. In fact, they prove an inequality of type $\alpha(H, t) \geq C H(t)$ for more general expansive interval maps and more general holes.

The set $K(t)$ is also related to the problem of diophantine approximation (see [11]): in fact, if one considers the set

$$
F_{t}:=\left\{x \in \mathbb{S}: x-m / 2^{n} \geq t / 2^{n} \text { for all but a finite number of } m, n\right\}
$$

of points which are not well approximable (in a suitable sense) by dyadic numbers, then, for any $t \in[0,1]$,

$$
\eta(t)=\text { H.dim } F_{t} .
$$

One important tool for the proof of Theorem 1 is a formula, due to Urbański [14, p. 305] which allows us to compute the value of $\eta(t)$ given the expansion in base $d$ of $t \in \mathcal{U}$. In order to state the result precisely, note that each real number $t \in(0,1]$ admits exactly one expansion $t=\epsilon_{1} \epsilon_{2} \ldots$ in base $d$ such that the sequence $\left(\epsilon_{n}\right)_{n \in \mathbb{N}}$ is not eventually zero. We shall call such an expansion the non-degenerate expansion of $t$. Let $t \in \mathcal{U}$ be a bifurcation parameter, and let $t=. \epsilon_{1} \epsilon_{2} \ldots$ be its non-degenerate expansion in base $d$. Then $\eta=\eta(t)$, the Hausdorff dimension of $K(t)$, is given by

$$
\eta=-\frac{\log \lambda}{\log d}
$$

where $\lambda$ is a root of the equation $P_{t}(\lambda)=1$, and $P_{t}(X)$ is the power series

$$
P_{t}(X):=\sum_{k=1}^{\infty}\left(d-1-\epsilon_{k}\right) X^{k} .
$$

Let us stress that this formula is only valid for $t \in \mathcal{U}$ (see Remark 12).

The other main ingredient in our approach is an explicit characterization of the expansions in base $d$ of the elements of $\mathcal{U}$. In particular, we will show (Proposition 5) that the connected components of the complement of $\mathcal{U}$ are naturally labeled by Lyndon words, that is, finite words which are minimal for the lexicographic order among their cyclic permutations. This also answers the question of Nilsson [11, §6], who asks for a characterization of the plateaux of the dimension function for $d>2$; in the case $d=2$, our characterization is essentially equivalent to that given in [11]. In fact, using this description of $\mathcal{U}$ we will recover, in a self-contained, elementary way, the main results of [14], using combinatorics on words rather than thermodynamic formalism (see Remark 17). Finally, the statement of Theorem 1 also holds for an alternative definition of local Hölder exponent (see Theorem 16).

Note that, without any reference to dynamics, one can ask whether there exist monotone, continuous functions $f:[0,1] \rightarrow[0,1]$ with the property that, for each $t \in[0,1]$, either $f$ is locally constant at $t$ or the local Hölder exponent of $f$ at $t$ equals $f(t)$. The functions $\eta(t)$, for varying $d$, provide an infinite number of such examples (hence this property does not determine the function uniquely).

Moreover, functions with this property seem to also appear in relation to other families of dynamical systems. In particular, if one considers the function $h(\theta):=h_{\text {top }}\left(f_{\theta}\right) / \log 2$, which expresses the (normalized) topological entropy of a real quadratic polynomial $f_{\theta}$, as 
a function of its external angle $\theta$ (or equivalently, as a function of its kneading sequence), then it is also expected that the local Hölder exponent of $h(t)$ at $t$ equals the value of the function $h(t)$ (see, for example, [4] and [6, §4]). Note that, in this case, the kneading series of [9] plays the same role as the power series $P_{t}(X)$ in this paper. However, in this case, the modulus of continuity at the smallest $t$, where $h(t)=0$ (the Feigenbaum parameter), is of order $1 /(\log (1 / x))$ (see, for example, $[12, \S 9.1])$. Another more complicated, nonmonotone case, where the local Hölder exponent is at least conjectured to equal the value of the function at every point, is given by the (normalized) core entropy function for quadratic polynomials introduced by W. Thurston (see, for example, [13]).

The underlying phenomenon, of which Theorem 1 and Corollary 2 provide quantitative statements in a specific case, seems to be that systems with low entropy are less stable than systems with high entropy, in the sense that a small perturbation leads to a large variation in entropy. It would be of great interest to investigate to what extent this phenomenon is universal.

\section{Word combinatorics and ordering}

Let $d \geq 2$ be fixed once and for all. We define the alphabet as the set $\mathcal{A}:=\{0,1, \ldots$, $d-1\}$, and (finite or infinite) sequences of elements of $\mathcal{A}$ will be called words. If $S, T \in$ $\mathcal{A}^{n}$ are finite words of equal length, we write $S<T$ to denote the lexicographical order; moreover, we shall extend the order to a partial order on the set of all finite words in the following way. If $S=\left(a_{1}, \ldots, a_{n}\right)$ and $T=\left(b_{1}, \ldots, b_{m}\right)$ are finite words, we write $S \ll T$ (and read $S$ is strongly less than $T$ ) if there exists an index $k \leq \min \{m, n\}$ such that $a_{i}=b_{i}$ for all $1 \leq i \leq k-1$ and $a_{k}<b_{k}$. For instance, 001 is strongly less than 01 but not strongly less than 00101 .

Definition 3. Let us define a finite word $S$ to be Lyndon if it is strongly less than all its proper suffixes: that is, if, for each decomposition $S=X Y$ into two non-empty words,

$$
S \ll Y .
$$

For instance, 011 is Lyndon because $011 \ll 11$ and $011 \ll 1$, but 01101 is not Lyndon, because 01 is a suffix of 01101 but 01101 is not strongly less than 01 . Note that words of one letter are Lyndon by definition.

We shall call $d$-rational a rational number which admits a finite expansion in base $d$, and denote the set of $d$-rational numbers contained in the interval $(0,1)$ by $\mathbb{Q}(d)$.

Definition 4. A $d$-rational number $r \in(0,1)$ is called Lyndon (for a given base $d$ ) if it admits a finite expansion $r=. \epsilon_{1} \ldots \epsilon_{m}$ in base $d$ such that the word $S=\epsilon_{1} \ldots \epsilon_{m}$ is Lyndon (note that such expansion is unique, because the Lyndon property implies $\epsilon_{m} \neq 0$ ).

We shall denote the set of Lyndon rational numbers in $(0,1)$ by $\mathbb{Q}_{\text {Lyn }}$. Note that the one letter word 0 is the only Lyndon word which does not correspond to a Lyndon rational number.

Finally, if $S=\epsilon_{1} \ldots \epsilon_{m}$ is a finite word and $x \in[0,1]$, we shall denote by

$$
S \cdot x:=\sum_{i=1}^{m} \epsilon_{i} d^{-i}+x d^{-m}
$$


the number whose expansion in base $d$ is $S$ followed by the expansion of $x$. The affine map $x \mapsto S \cdot x$ is an inverse to $g^{m}$ and is uniformly contracting with derivative $d^{-m}$.

Lyndon words appear in several contexts in combinatorics: for a reference, see, for example, [8, p. 64]. Another equivalent definition given in the literature is that a word $S$ is Lyndon if it is the smallest among all its cyclic permutations: that is, if

$$
S<Y X
$$

whenever $S=X Y$ is a decomposition of $S$ into two non-empty words (for the equivalence, cf. Lemma 9).

\section{The bifurcation set}

Let us start by considering the function $t \mapsto K(t)$ as a function into sets. We shall call a parameter $t \in[0,1]$ stable if the function $t \mapsto K(t)$ is locally constant at $t$ : that is, if there exists $\epsilon>0$ such that the equality

$$
K\left(t^{\prime}\right)=K(t)
$$

holds for each $t^{\prime} \in[t-\epsilon, t+\epsilon]$. We call such a set of parameters the stable set. A parameter which is not stable will be called a bifurcation parameter, and the set of all bifurcation parameters will be called bifurcation set and denoted by $\mathcal{U}$.

The set $\mathcal{U}$ is closed with no interior, and has the characterization given by

$$
\mathcal{U}=\left\{t \in[0,1]: g^{k}(t) \notin(0, t) \forall k \geq 0\right\}
$$

(for a proof, see Lemma 6). The main goal of this section is to characterize all connected components of the complement of $\mathcal{U}$; we shall see that they are naturally labeled by Lyndon rational numbers.

Let us define, for each $d$-rational $r \in \mathbb{Q}_{(d)}$, the interval

$$
I_{r}:=\left(. \epsilon_{1} \ldots \epsilon_{m}, \overline{\epsilon_{1} \ldots \epsilon_{m}}\right)
$$

with left endpoint $r$ and right endpoint the rational number with periodic base- $d$ expansion $\epsilon_{1} \ldots \epsilon_{m}$. For instance, in the case $d=2$, if $r=1 / 4=.01$, then $. \overline{01}=1 / 3$ and hence $I_{1 / 4}=(1 / 4,1 / 3)$. Note also that $I_{1 / 2}=(1 / 2,1)$.

PROPOSITION 5. The connected components of the complement of $\mathcal{U}$ are parametrized by Lyndon rational numbers. Indeed, we have the identities

$$
[0,1] \backslash \mathcal{U}=\bigsqcup_{r \in \mathbb{Q}_{\mathrm{Lyn}}} I_{r}=\bigcup_{r \in \mathbb{Q}_{(d)}} I_{r} .
$$

The proposition will follow from the following lemmata.

LEMMA 6. Let $t \in[0,1)$. Then the following are equivalent:

(1) the element $t$ belongs to $K(t)$; and

(2) $t$ is a bifurcation parameter. 
Proof of Lemma 6. If $t \in K(t)$, then, for each $t^{\prime}>t$, the element $t$ belongs to $K(t) \backslash K\left(t^{\prime}\right)$, proving (2).

If, instead, $t \notin K(t)$, then let $t^{\prime}:=\inf \{x \in[t, 1]: x \in K(t)\}>t$ (the inequality is strict since $K(t)$ is closed). We claim that $K\left(t^{\prime}\right)=K(t)$; indeed, if $x \in K(t)$ then, for each $k$, we have $g^{k}(x) \in K(t) \subset\left[t^{\prime}, 1\right]$, and hence $x \in K\left(t^{\prime}\right)$. Moreover, let us show that there exists $\epsilon>0$ such that, for $t^{\prime} \in(t-\epsilon, t)$, we have $K\left(t^{\prime}\right)=K(t)$. If not, then there would be a sequence of parameters $t_{n} \rightarrow t, t_{n}<t$ and a sequence of elements $x_{n} \in K\left(t_{n}\right) \backslash K(t)$. By taking forward images of $x_{n}$, we would then get a sequence $y_{n}=g^{k_{n}}\left(x_{n}\right) \in K\left(t_{n}\right) \cap\left[t_{n}, t\right]$ : this would imply that $y_{n} \rightarrow t$ and

$$
g^{k}\left(y_{n}\right) \in\left[t_{n}, 1\right]
$$

for each $k$ and $n$. Thus, since $g$ is continuous on $\mathbb{S}$, we would have $g^{k}(t) \in[t, 1]$, which contradicts the fact that $t \notin K(t)$.

LeMma 7. For each $d$-rational $r \in \mathbb{Q}_{(d)}$, the interval $I_{r}$ is contained in the stable set $[0,1] \backslash \mathcal{U}$.

Proof. Indeed, if $r=. \epsilon_{1} \ldots \epsilon_{m}$, then the map $g^{m}$ is uniformly expanding of derivative $d^{m}$, it has $\bar{r}=. \overline{\epsilon_{1} \ldots \epsilon_{m}}$ as its fixed point and maps $(r, \bar{r})$ onto $(0, \bar{r})$. Thus, if $x \in(r, \bar{r})$, then $\left|g^{m}(x)-\bar{r}\right|>|x-\bar{r}|$, and hence $g^{m}(x) \in(0, x)$ and $x \notin \mathcal{U}$.

Lemma 8. Let $x \notin \mathcal{U}$. Then $x$ belongs to some interval $I_{r}$ with $\partial I_{r} \subseteq \mathcal{U}$.

Proof. Let $x \notin \mathcal{U}$, and $k \geq 1$ be the minimum value such that

$$
g^{k}(x) \in(0, x) .
$$

Let $x=. \epsilon_{1} \epsilon_{2} \ldots$ be the non-degenerate expansion of $x$, let $S_{k}:=\epsilon_{1} \ldots \epsilon_{k}$ denote its truncation and write $r:=S_{k} \cdot 0=\epsilon_{1} \ldots \epsilon_{k}$. Note that the map $g^{k}$ is an orientationpreserving bijection from $J_{k}:=S_{k} \cdot(0,1]$ onto $(0,1]$ with derivative $d^{k}$, and $\bar{r}:=$ $\overline{\epsilon_{1} \ldots \epsilon_{k}}$ is its fixed point. Now note that, by construction, $x$ belongs to $J_{k}$; moreover, if $x \geq \bar{r}$, then $g^{k}(x)=d^{k}(x-\bar{r})+\bar{r} \geq x$, which contradicts equation (7). Thus, $x$ belongs to $I_{r}:=(r, \bar{r})$, proving the first part of the claim.

Moreover, we claim that, for each $h=\{1, \ldots, k-1\}$,

$$
g^{h}\left(J_{k}\right) \subseteq(\bar{r}, 1)
$$

which implies that both $r$ and $\bar{r}$ belong to $\mathcal{U}$. Thus $\partial I_{r} \subseteq \mathcal{U}$, as required. To prove equation (8), let us pick $y \in J_{k}$; if $y \geq \bar{r}$, then

$$
g^{h}(y)=g^{h}(x)+d^{h}(y-x)>x+(y-x)=y \geq \bar{r} .
$$

Now, if there exists $y \in J_{k} \cap(0, \bar{r})$ such that $g^{h}(y)<\bar{r}$, then, by the intermediate value theorem, there must exist $z \in J_{k} \cap(0, \bar{r})$ such that $g^{h}(z)=\bar{r}$, and hence $g^{k}(z)=$ $g^{k-h}(\bar{r}) \geq \bar{r}$, by the previous observation (equation (9) with $k-h$ instead of $h$ ). However, this is contradictory because $g^{k}(z) \in g^{k}\left(J_{k} \cap(0, \bar{r})\right)=(0, \bar{r})$.

LeMmA 9. Let $S=\epsilon_{1} \ldots \epsilon_{m} \in \mathcal{A}^{m}$ be a word with $\epsilon_{m} \neq 0$, and $r=. \epsilon_{1} \ldots \epsilon_{m}$ the associated $d$-rational. Then the following are equivalent: 
(1) $I_{r}$ belongs to $\mathcal{U}$; and

(2) S is a Lyndon word.

Proof. If $S$ is Lyndon, then, for each $h \in\{1, \ldots, m-1\}$,

$$
g^{h}(r)=. \epsilon_{h+1} \ldots \epsilon_{m} \overline{0}>. \epsilon_{1} \ldots \epsilon_{m} \overline{0}=r
$$

and, similarly, $g^{h}(\bar{r})>\bar{r}$. Thus the endpoints of $I_{r}$ belong to $\mathcal{U}$. Conversely, if $r \in \mathcal{U}$, then, for each $h \in\{1, \ldots, m-1\}$,

$$
. \epsilon_{1} \ldots \epsilon_{m} \overline{0}=r \leq g^{h}(r)=. \epsilon_{h+1} \ldots \epsilon_{m} \overline{0}
$$

and hence $\epsilon_{1} \ldots \epsilon_{m} \ll \epsilon_{h+1} \ldots \epsilon_{m}$ unless $\epsilon_{h+1} \ldots \epsilon_{m}$ is a prefix of $\epsilon_{1} \ldots \epsilon_{m}$ and $\epsilon_{m-h+1} \ldots \epsilon_{m}$ is all zeros, which is not possible since $\epsilon_{m} \neq 0$, by hypothesis.

Proof of Proposition 5. It is easy to prove that the complement of $\mathcal{U}$ is open: namely, if $t \notin \mathcal{U}$, then, by Lemma $6(1)$, there exists $k \geq 0$ such that $g^{k}(t) \in(0, t)$, and such a condition is open in $t$. From Lemmas 8,9 and 7 , respectively, we have the chain of inclusions

$$
[0,1] \backslash \mathcal{U} \subseteq \bigcup_{\partial I_{r} \subseteq \mathcal{U}} I_{r} \subseteq \bigcup_{r \in \mathbb{Q}_{(d)}} I_{r} \subseteq[0,1] \backslash \mathcal{U},
$$

and thus equality must hold. Note, also, that two intervals $I_{r}$ whose endpoints lie in $\mathcal{U}$ may not overlap, and hence their union must be disjoint. Moreover, by Lemma 9, the set of rationals $r$, for which $\partial I_{r} \subseteq \mathcal{U}$, coincides with the set $\mathbb{Q}_{\text {Lyn }}$ of Lyndon rationals, and hence we get

$$
[0,1] \backslash \mathcal{U}=\bigsqcup_{r \in \mathbb{Q}_{\text {Lyn }}} I_{r}=\bigcup_{r \in \mathbb{Q}_{(d)}} I_{r} .
$$

As a consequence, the complement of $\mathcal{U}$ contains a right neighborhood of any $d$-rational, and hence $\mathcal{U}$ has no interior.

\section{Structure and dimension of $K(t)$}

In this chapter, we show that the set $K(t)$ has a countable Markov partition, which we can easily describe, and can be used to compute the Hausdorff dimension of $K(t)$. This gives an alternative proof of Urbański's formula [14, p. 305].

Proposition 10. Let $d \geq 2$, and $t \in \mathcal{U}$ be a bifurcation parameter with non-degenerate base-d expansion $t=. \epsilon_{1} \epsilon_{2} \ldots$ Then $\eta=\eta(t)$, the Hausdorff dimension of $K(t)$, is given by

$$
\eta=-\frac{\log \lambda}{\log d}
$$

where $\lambda$ is a root of the equation

$$
P_{t}(\lambda)=1
$$

and $P_{t}(X)$ is the power series

$$
P_{t}(X):=\sum_{k=1}^{\infty}\left(d-1-\epsilon_{k}\right) X^{k} .
$$


Note that the series $P_{t}(X)$ always converges inside the unit disk and, by the intermediate value theorem, equation (10) has exactly one root in the interval $(0,1]$. Whenever $t$ has a purely periodic expansion of period $p$, the series $P_{t}(X)$ becomes a rational function and $\lambda=d^{-\eta(t)}$ is the root of a polynomial of degree $p$.

As an example, in the case $d=2$, if $t=\overline{.001}=1 / 7$, then

$$
P_{t}(X)=X+X^{2}+X^{4}+X^{5}+\cdots=\frac{X+X^{2}}{1-X^{3}}
$$

so $\lambda=2^{-\eta(t)}$ is a root of $P_{t}(\lambda)=1$, that is, satisfies $\lambda^{3}+\lambda^{2}+\lambda-1=0$.

Let $t \in(0,1]$, and $t=\epsilon_{1} \epsilon_{2} \ldots$ be its non-degenerate expansion in base $d$. For each $k \geq 1$ and $s \in \mathcal{A}$, define the word

$$
S_{k, s}(t):=\epsilon_{1} \ldots \epsilon_{k-1} s
$$

and consider the set of words

$$
\Sigma(t):=\left\{S_{k, s}(t): \epsilon_{k}<s\right\} .
$$

The following proposition characterizes precisely the elements which belong to $K(t)$ in terms of the set $\Sigma(t)$.

Proposition 11. Let $t \in \mathcal{U}$ be a bifurcation parameter. Then we have the identity

$$
K(t)=\{t\} \cup \bigsqcup_{S \in \Sigma(t)} S \cdot K(t) .
$$

That is, an element belongs to $K(t)$ if and only if its (non-degenerate) expansion in base $d$ is a concatenation of words in $\Sigma(t)$.

Proof. Let $x \in K(t)$. Then, by definition, $x \in[t, 1]$, and hence either $x=t$ or the expansion of $t$ starts with $S_{k, s}$, where $k$ is the first digit for which the expansions of $t$ and $x$ differ, and $s$ is the $k$ th digit of $x$, which must be larger than the $k$ th digit of $t$. Hence $x=S_{k, s} \cdot y$ with $y \in[0,1]$ and, since $K(t)$ is forward invariant, $y=g^{k}(x)$ also belongs to $K(t)$, so $x \in S_{k, s} \cdot K(t)$.

Conversely, let $x=S \cdot y$ with $S \in \Sigma(t)$ and $y \in K(t)$. We have to prove that $g^{h}(x) \in$ $[t, 1]$ for each $h \geq 0$. If $h \geq k$, then $g^{h}(x)=g^{h-k}(y) \in[t, 1]$ and the claim is proven. On the other hand, fix $h \in\{0, \ldots, k-1\}$ and compare the expansions of $g^{h}(x)$ and $g^{h}(t)$. Since the expansion of $g^{h}(x)$ begins with.$\epsilon_{h+1} \ldots \epsilon_{k-1} s$ and the expansion of $g^{h}(t)$ begins with.$\epsilon_{h+1} \ldots \epsilon_{k-1} \epsilon_{k}$ and $s>\epsilon_{k}$,

$$
g^{h}(x) \in\left[g^{h}(t), 1\right] \subseteq[t, 1],
$$

where, in the last inequality, we used that $t$ belongs to $\mathcal{U}$. Thus the claim is proven.

Proof of Proposition 10. Consider the set $\widetilde{K}(t):=\left\{x \in K(t): g^{k}(x) \neq t \forall k \geq 0\right\}$. Since $K(t)$ and $\widetilde{K}(t)$ differ by a countable set of preimages of $t$, their Hausdorff dimension is the same; moreover, by Proposition 11,

$$
\widetilde{K}(t)=\bigsqcup_{S \in \Sigma(t)} S \cdot \widetilde{K}(t) .
$$




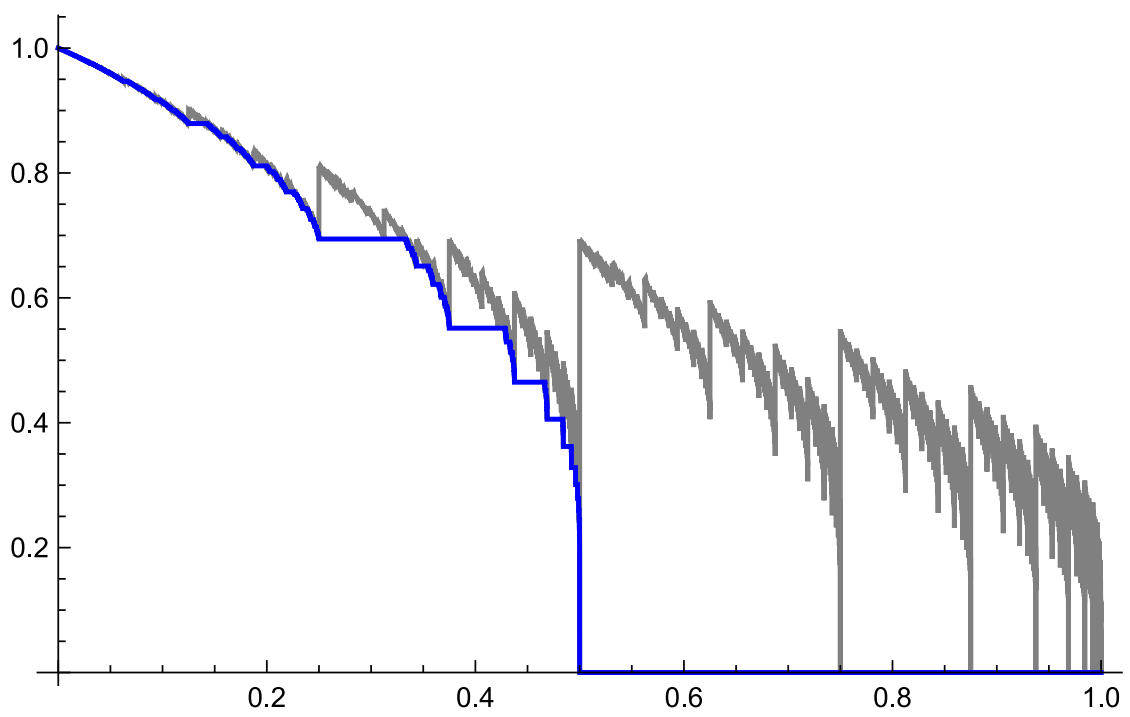

FIGURE 2. The functions $\zeta(t)$ and $\eta(t)$ for $d=2$.

The set $\widetilde{K}(t)$ is thus the attractor of a countable iterated function system; each map $x \mapsto$ $S_{k, s} \cdot x$ is an affine map of derivative $d^{-k}$ and, moreover, by construction, all the images $S_{k, s} \cdot \widetilde{K}(t)$ are disjoint and satisfy the open set condition $[\mathbf{5}, \mathbf{1 0}]$. Hence the Hausdorff dimension $\eta$ of $\widetilde{K}(t)$ is determined implicitly by the formula

$$
1=\sum_{S_{k, s} \in \Sigma(t)} d^{-k \eta}
$$

which, since by definition of $\Sigma(t)$ for each $k$ there are $d-1-\epsilon_{k}$ values of $s$, can also be written as

$$
1=\sum_{k=1}^{\infty}\left(d-1-\epsilon_{k}\right) d^{-k \eta} .
$$

Thus taking $X=d^{-\eta}$ yields the claim.

Remark 12. Note that the hypothesis $t \in \mathcal{U}$ in Proposition 10 is essential. Actually, one can define for any $t \in[0,1]$ the function $\zeta(t)=-\log \lambda / \log d$, where $\lambda$ is the unique real, positive root of the equation $P_{t}(X)=1$. Then the function $\zeta(t)$ is no longer continuous, but for any $t \in[0,1]$ one has the relation (see Figure 2)

$$
\eta(t)=\min \{\zeta(s), 0 \leq s \leq t\} .
$$

4.1. The local Hölder exponent. Let us recall that a function $f: I \rightarrow \mathbb{R}$ on an interval $I$ is called Hölder continuous of exponent $\alpha$ if there exists a constant $C>0$ such that, for each $x, y \in I$,

$$
|f(x)-f(y)| \leq C|x-y|^{\alpha} .
$$

Given $t \in I$, we define the local Hölder exponent of $f$ at $t$ to be

$$
\alpha(f, t):=\liminf _{t^{\prime} \rightarrow t} \frac{\log \left|f(t)-f\left(t^{\prime}\right)\right|}{\log \left|t-t^{\prime}\right|} .
$$


The goal of this section is to establish Theorem 1, namely, that

$$
\alpha(\eta, t)=\eta(t)
$$

for any $t \in \mathcal{U}$. Let us start with some preliminary remarks.

First let us note that, for $t \in((d-1) / d, 1]$, the set $K(t)=\{0\}$ is one point so $\eta(t)=0$ and there is nothing to prove. Therefore, we shall assume $t \in[0,(d-1) / d]$. In this case, let us note that $P_{t}(X)=s X+\sum_{k=2}^{\infty}\left(d-1-\epsilon_{k}\right) X^{k}$ with $s \geq 1$, and hence the function $P_{t}(x)$ is strictly increasing on $[0,1)$. Moreover, $P_{t}(0)=0$ and $\lim _{x \rightarrow 1^{-}} P_{t}(x)>1$ unless $t=(d-1) / d$ (in which case $\left.P_{t}(x)=x\right)$. Thus, for each $t \in[0,(d-1) / d]$, the equation $P_{t}(x)=1$ has a unique solution $\lambda \in(0,1]$, which we will denote by $\lambda(t)$. Note also that, for each $x \in(0,1)$,

$$
1 \leq P_{t}^{\prime}(x) \leq \frac{d}{(1-x)^{2}},
$$

and hence $\lambda(t)$ is always a simple root of $P_{t}(X)$.

If $t \in[0,1]$, we shall denote the $k$ th digit of the non-degenerate expansion of $t$ by $\epsilon_{k}(t)$. Moreover, if $t_{1}, t_{2} \in[0,1]$, let us define $m\left(t_{1}, t_{2}\right)$ to be the length of the longest common prefix in the expansions of $t_{1}$ and $t_{2}$ : namely,

$$
m\left(t_{1}, t_{2}\right):=\sup \left\{k \geq 0: \epsilon_{h}\left(t_{1}\right)=\epsilon_{h}\left(t_{2}\right) \forall h \in\{1, \ldots, k\}\right\} .
$$

LEMMA 13. For each $t_{0}>0$, there exists a constant $C_{1}>0$ such that, for each $t_{1}, t_{2} \in$ $\mathcal{U} \cap\left[t_{0}, 1\right]$

$$
C_{1} d^{-m\left(t_{1}, t_{2}\right)} \leq\left|t_{1}-t_{2}\right| \leq d^{-m\left(t_{1}, t_{2}\right)}
$$

Proof. Let $m:=m\left(t_{1}, t_{2}\right)$; the upper bound is given by $\left|t_{1}-t_{2}\right|=d^{-m} \mid g^{m}\left(t_{1}\right)-$ $g^{m}\left(t_{2}\right) \mid \leq d^{-m}$. To get the lower bound, first note that, since $K\left(t_{0}\right) \subseteq\left[t_{0}, 1\right]$ and $K\left(t_{0}\right)$ is forward invariant by $g$,

$$
K\left(t_{0}\right) \subseteq g^{-1}\left(K\left(t_{0}\right)\right) \subseteq g^{-1}\left(\left[t_{0}, 1\right]\right)=\bigcup_{k=0}^{d-1} I_{k},
$$

where $I_{k}:=\left[\left(t_{0}+k\right) / d,(1+k) / d\right]$. Now, by definition of $m$, the two points $u_{1}:=g^{m}\left(t_{1}\right)$ and $u_{2}:=g^{m}\left(t_{2}\right)$ belong to two different intervals $I_{k}$, and thus $\left|t_{1}-t_{2}\right|=d^{-m}\left|u_{1}-u_{2}\right| \geq$ $t_{0} / d$, which gives the lower bound with $C_{1}:=t_{0} / d$.

We are now ready to prove the main theorem stated in the introduction.

Proof of Theorem 1. Monotonicity of $\eta(t)$ is immediate from the definition, while continuity follows from Rouché's theorem. Indeed, let $t \in \mathcal{U} \cap[0,(d-1) / d]$ and suppose $\lambda=\lambda(t)<1$. Then, $\lambda(t)$ is a simple root of $P_{t}(X)$, and $P_{t^{\prime}}(X)$ converges uniformly on compact sets to $P_{t}(X)$ as $t^{\prime} \rightarrow t$. Hence the root $\lambda\left(t^{\prime}\right)$ converges to $\lambda(t)$. Now suppose that $\lambda(t)=1$, which implies that $t=(d-1) / d$. Fix $\delta \in(0,1)$. Then $P_{t}(X)-1=X-1$ has no roots in the strip $S_{\delta}=\{x+i y: 0 \leq x \leq 1-\delta,|y| \leq \delta\}$, and hence, by Rouché's theorem, $P_{t^{\prime}}(X)$ also has no roots in $S_{\delta}$ for $t^{\prime}$ sufficiently close to $t$. Hence $\lambda\left(t^{\prime}\right) \geq \delta$. This proves $\lambda\left(t^{\prime}\right) \rightarrow 1$ as $t^{\prime} \rightarrow t$. Since $\eta(t)=-\log \lambda(t) / \log d$, continuity of $\lambda(t)$ implies continuity of $\eta(t)$. 
Let us now estimate the modulus of continuity of $\eta(t)$. First note that, since $\eta(t)=$ $-\log \lambda(t) / \log d$ and the function $h(x):=-\log x / \log d$ is bi-Lipschitz on $[1 / d, 1]$, it is equivalent to prove the claim for $\lambda(t)$. Let $t_{1}, t_{2} \in \mathcal{U} \cap[0,(d-1) / d]$. To simplify notation, we denote $\lambda_{1}:=\lambda\left(t_{1}\right), \lambda_{2}:=\lambda\left(t_{2}\right)$, and also $P_{1}(X):=P_{t_{1}}(X), P_{2}(X):=P_{t_{2}}(X)$ and suppose that $\lambda_{1}, \lambda_{2}<1$.

Now, using that $P_{2}\left(\lambda_{2}\right)=P_{1}\left(\lambda_{1}\right)=1$ and applying Lagrange's theorem,

$$
P_{1}\left(\lambda_{1}\right)-P_{2}\left(\lambda_{1}\right)=P_{2}\left(\lambda_{2}\right)-P_{2}\left(\lambda_{1}\right)=P_{2}^{\prime}(\xi)\left(\lambda_{2}-\lambda_{1}\right)
$$

for some $\xi \in\left[\lambda_{1}, \lambda_{2}\right]$. On the other hand, by writing out the power series we get

$$
P_{1}\left(\lambda_{1}\right)-P_{2}\left(\lambda_{1}\right)=\lambda_{1}^{m+1} R\left(t_{1}, t_{2}\right),
$$

where $R\left(t_{1}, t_{2}\right):=\left(\epsilon_{m+1}\left(t_{2}\right)-\epsilon_{m+1}\left(t_{1}\right)\right)+\sum_{j=m+2}^{\infty}\left(\epsilon_{j}\left(t_{2}\right)-\epsilon_{j}\left(t_{1}\right)\right) \lambda_{1}^{j-m-1}$ and $m=$ $m\left(t_{1}, t_{2}\right)$. By comparing the two previous equations we get

$$
\left|\lambda_{1}-\lambda_{2}\right|=\lambda_{1}^{m+1} \frac{\left|R\left(t_{1}, t_{2}\right)\right|}{P_{2}^{\prime}(\xi)} \leq \lambda_{1}^{m+1} \frac{d}{1-\lambda_{1}} .
$$

Hence, by combining this with the upper bound for $\left|t_{1}-t_{2}\right|$ given by equation (13), we have the following upper bound for the modulus of continuity. For each $t \in \mathcal{U} \cap$ $(0,(d-1) / d)$, there exists $C_{2}>0$ such that

$$
\left|\lambda_{1}-\lambda_{2}\right| \leq C_{2}\left|t_{1}-t_{2}\right|^{-\log \lambda_{1} / \log d}=C_{2}\left|t_{1}-t_{2}\right|^{\eta\left(t_{1}\right)}
$$

for each $t_{1}, t_{2} \in \mathcal{U}$ sufficiently close to $t$. Since $\lambda(t)$ is constant on the complement of $\mathcal{U}$, the above upper bound actually works for any $t_{1}, t_{2}$ close to $t$, proving that

$$
\alpha(\eta, t) \geq \eta(t)
$$

for each $t \in \mathcal{U}$.

For the lower bound, let us pick $t \in \mathcal{U}$. Now, by Lemma 14, there exists a sequence $t_{n} \rightarrow t$ with $t_{n} \neq t$ such that, for each $k$ and each $n$, we either have $\epsilon_{k}(t) \leq \epsilon_{k}\left(t_{n}\right)$ or we have the reverse inequality. In both cases, $R\left(t, t_{n}\right)$ is a power series in $\lambda(t)$, the coefficients of which are all are integers and have the same sign. Hence $\left|R\left(t, t_{n}\right)\right| \geq 1$ and

$$
\left|\lambda(t)-\lambda\left(t_{n}\right)\right|=\lambda(t)^{m+1} \frac{\left|R\left(t, t_{n}\right)\right|}{P_{2}^{\prime}(\xi)} \geq \lambda(t)^{m+1} \cdot \frac{(1-\xi)^{2}}{d} \geq C_{3}\left|t-t_{n}\right|^{\eta(t)},
$$

where $C_{3}:=\lambda(t) \inf _{\tau \in\left[t, t_{n}\right]}\left((1-\lambda(\tau))^{2}\right) / d$. This proves that the lower bound $\alpha(\eta, t) \leq$ $\eta(t)$.

LEMMA 14. Let $t \in \mathcal{U}$. If $t$ is not a d-rational, then there exists a sequence $\left(t_{n}\right)$ of elements of $\mathcal{U}$ such that $t_{n} \rightarrow t, t_{n}>t$ for any $n$, and

$$
\epsilon_{k}(t) \leq \epsilon_{k}\left(t_{n}\right) \quad \text { for all } k, n \text {. }
$$

If $t$ is a d-rational, then there exists a sequence $\left(t_{n}\right)$ of elements of $\mathcal{U}$ such that $t_{n} \rightarrow t$, $t_{n}<t$ for any $n$, and

$$
\epsilon_{k}\left(t_{n}\right) \leq \epsilon_{k}(t) \quad \text { for all } k, n
$$


Proof. Let $t \in \mathcal{U}$ not a $d$-rational, and let $t=. \epsilon_{1} \epsilon_{2} \ldots$ be its (non-degenerate) expansion in base $d$. For each $n$, let us define

$$
t_{n}:=. \epsilon_{1} \ldots \epsilon_{n}(d-1)^{\infty} .
$$

By construction, $t_{n}>t, t_{n} \rightarrow t$, and $\epsilon_{k}\left(t_{n}\right) \geq \epsilon_{k}(t)$ for each $k$. We need to check that $t_{n} \in \mathcal{U}$. Let us consider $g^{r}\left(t_{n}\right)$ and compare it with $t_{n}$. If $r \geq n$, then $g^{r}\left(t_{n}\right)=0 \notin\left(0, t_{n}\right)$, as required. If, instead, $r<n$, then $g^{r}\left(t_{n}\right)=. \epsilon_{r+1} \ldots \epsilon_{n}(d-1)^{\infty}$. Since $t \in \mathcal{U}$, then $g^{r}(t)=. \epsilon_{r+1} \ldots \epsilon_{n} \ldots \geq t=. \epsilon_{1} \ldots \epsilon_{n} \ldots$, and hence, if you set $S:=\epsilon_{1} \ldots \epsilon_{n}$ and $S_{0}:=\epsilon_{r+1} \ldots \epsilon_{n}$, either $S_{0} \gg S$ or $S_{0}$ is a prefix of $S$. In the first case, $g^{r}\left(t_{n}\right) \geq t_{n}$ as required; in the second case, $S_{0}(d-1)^{n-r} \geq S$, so $g^{r}\left(t_{n}\right)=. S_{0}(d-1)^{\infty} \geq . S(d-1)^{\infty}=$ $t_{n}$, as required.

Let us now deal with the case where $t$ is a $d$-rational, and let $t=. \epsilon_{1} \ldots \epsilon_{k}(d-1)^{\infty}$ be its non-degenerate expansion, which we can take so that $\epsilon_{1} \neq d-1$ and $\epsilon_{k} \neq d-1$. We claim that the number $t_{n}$ with base- $d$ expansion

$$
t_{n}=\overline{\epsilon_{1} \ldots \epsilon_{k}(d-1)^{n}}
$$

satisfies the claim. Clearly, $t_{n}<t$ and $t_{n} \rightarrow t$, while $\epsilon_{k}\left(t_{n}\right) \leq \epsilon_{k}(t)$ for any $k$. We need to prove that $t_{n} \in \mathcal{U}$. Given $r$, consider $g^{r}\left(t_{n}\right)$ : either the first digit of $g^{r}\left(t_{n}\right)$ is $(d-1)$, which implies $g^{r}\left(t_{n}\right) \notin\left(0, t_{n}\right)$ as $\epsilon_{1} \neq d-1$, or $g^{r}\left(t_{n}\right)$ is of the form $g^{r}\left(t_{n}\right)=. \epsilon_{r+1} \ldots \epsilon_{k}$ $(d-1)^{n} \ldots$ Then, if $S:=\epsilon_{1} \ldots \epsilon_{n}$ and $S_{0}:=\epsilon_{r+1} \ldots \epsilon_{k}$, we have either $S \ll S_{0}$ or $S_{0}$ is a prefix of $S$. If $S_{0}$ is a prefix of $S$, then one can write $S=S_{0} S_{1}$, where $S_{1}$ is some non-empty word, and either $S_{1} \ll(d-1)^{n}$ or $S_{1}$ is of the form $(d-1)^{a}$ for some $a \geq 1$, which contradicts the fact that $\epsilon_{k} \neq d-1$.

Now we shall show that the function $\eta$ is not Hölder continuous at $t_{*}=1-1 / d$ (which is the smallest $t$ such that $\eta(t)=0$ ). In fact, the modulus of continuity of $\eta$ at $t_{*}$ is given by the function

$$
\omega(x):=\frac{\log \log (1 / x)}{\log (1 / x)},
$$

as shown in the following proposition.

Proposition 15. We have the limit

$$
\lim _{t \rightarrow t_{*}^{-}} \frac{\eta(t)-\eta\left(t_{*}\right)}{\omega\left(t_{*}-t\right)}=1
$$

Proof. To begin, we shall give a precise estimate of $\eta\left(t_{n}\right)$, where $t_{n}:=t_{*}-1 / d^{n}$. It is easy to check that $t_{n} \in \mathcal{U}$ for all $n \geq 2$ and $P_{t_{n}}(X)=X+X^{n}$. In order to locate the unique positive solution $\lambda_{n}$ of the equation $P_{t_{n}}(X)=1$, let us observe that, for any fixed $\alpha>0$,

$$
P_{t_{n}}\left(1-\alpha \frac{\log n}{n}\right)=1-\alpha \frac{\log n}{n}+\frac{1}{n^{\alpha}}\left[1+O\left(\frac{\log ^{2} n}{n}\right)\right] \text { as } n \rightarrow \infty .
$$

Therefore, using the above formula for $\alpha=1$, we get that there is $n_{0}$ such that $P_{t_{n}}(1-$ $(\log n / n))<1$ for all $n \geq n_{0}$. On the other hand, for any $\alpha<1$, there is $n_{1}=n_{1}(\alpha)$ such that $P_{t_{n}}(1-\alpha(\log n / n))>1$ for all $n \geq n_{1}$. This means that as $n \rightarrow \infty$ we have $\lambda_{n}=$ $1-(\log n / n)[1+o(1)]$ and

$$
\eta\left(t_{n}\right)=-\frac{\log \lambda_{n}}{\log d}=\frac{\log n}{n \log d}[1+o(1)] .
$$


Recalling that $\log \left(t_{*}-t_{n}\right)=-n \log d$, we see that the modulus of continuity of $\eta$ at $t_{*}$ cannot be smaller than $\omega$ : indeed,

$$
\lim _{n \rightarrow+\infty} \frac{\eta\left(t_{n}\right)-\eta\left(t_{*}\right)}{\omega\left(t_{*}-t_{n}\right)}=1 .
$$

On the other hand, if $t_{n} \leq t \leq t_{n+1}$ then, using the monotonicity of $\eta(t)$ and $\omega(t)$ and the fact that $\omega\left(t_{*}-t_{n}\right) / \omega\left(t_{*}-t_{n+1}\right) \rightarrow 1$, we get

$$
\lim _{t \rightarrow t_{*}^{-}} \frac{\eta(t)-\eta\left(t_{*}\right)}{\omega\left(t_{*}-t\right)}=1 .
$$

Let us now turn to the proof of Corollary 2 stated in the introduction, namely, the extension of these results to general expanding circle maps.

Proof of Corollary 2. Since $f$ is an expanding $C^{1}$ map, then it is conjugate to the linear map $g(x):=d x \bmod 1$, and the conjugacy is Hölder continuous: that is, there exists a Hölder continuous homeomorphism $\varphi: \mathbb{S} \rightarrow \mathbb{S}$ with Hölder continuous inverse such that $\varphi \circ f=g \circ \varphi$ (see, for example, [2, §II.2]). Since topological entropy is invariant by conjugacy,

$$
h_{\text {top }}\left(\left.f\right|_{K_{f}(t)}\right)=h_{\text {top }}\left(\left.g\right|_{K(\tau)}\right),
$$

where $\tau=\varphi(t)$. Then, since $\varphi$ and its inverse are Hölder continuous, there exists $C>0$ such that, for any $t$,

$$
C^{-1} \leq \liminf _{t^{\prime} \rightarrow t} \frac{\log \left|\tau^{\prime}-\tau\right|}{\log \left|t^{\prime}-t\right|} \leq \limsup _{t^{\prime} \rightarrow t} \frac{\log \left|\tau^{\prime}-\tau\right|}{\log \left|t^{\prime}-t\right|} \leq C,
$$

where $\tau^{\prime}=\varphi\left(t^{\prime}\right)$. Let us denote $H_{f}(t)=h_{\text {top }}\left(\left.f\right|_{K_{f}(t)}\right)$ and $H_{g}(\tau)=h_{\text {top }}\left(\left.g\right|_{K(\tau)}\right)$. Then, putting the estimates together, we get

$$
\begin{aligned}
\alpha\left(H_{f}, t\right) & =\liminf _{t^{\prime} \rightarrow t} \frac{\log \left|H_{f}\left(t^{\prime}\right)-H_{f}(t)\right|}{\log \left|t^{\prime}-t\right|} \geq C \liminf _{\tau^{\prime} \rightarrow \tau} \frac{\log \left|H_{g}\left(\tau^{\prime}\right)-H_{g}(\tau)\right|}{\log \left|\tau^{\prime}-\tau\right|} \\
& =C H_{g}(\tau)=C H_{f}(t)
\end{aligned}
$$

and, similarly, for the lower bound. Therefore we get, for any $t \in \mathcal{U}^{\prime}=\varphi(\mathcal{U})$, the bounds

$$
C^{-1} H_{f}(t) \leq \alpha\left(H_{f}, t\right) \leq C H_{f}(t) .
$$

As a consequence, if $t_{\star}:=\sup \left\{t: H_{f}(t)>0\right\}$, we have $H_{f}\left(t_{\star}\right)=0$, so $\alpha\left(H_{f}, t_{\star}\right)=0$ and hence $H_{f}$ is not locally Hölder continuous at $t_{\star}$.

Note that an alternative way to define the local Hölder exponent of $f$ at $t$ is as the supremum of all values $s$ for which $f$ is Hölder continuous of exponent $s$ on some neighborhood of $t$ : that is, as

$$
\widetilde{\alpha}(f, t):=\sup \left\{s: \lim _{\epsilon \rightarrow 0} \sup _{\substack{x, y \in B(t, \epsilon) \\ x \neq y}} \frac{|f(x)-f(y)|}{|x-y|^{s}}<\infty\right\},
$$

where $B(t, \epsilon)$ is the open ball of radius $\epsilon$ and center $t$. Note that $\widetilde{\alpha}(f, t)>0$ if and only if $f$ is locally Hölder continuous at $t$. While, in general, $\widetilde{\alpha}(f, t) \leq \alpha(f, t)$, the two quantities need not be the same. However, in our case the same argument as in the proof of Theorem 1 shows the following theorem. 
THEOREM 16. For each $t \in \mathcal{U}$,

$$
\widetilde{\alpha}(f, t)=\alpha(f, t)=\eta(t) .
$$

Proof. Since $\widetilde{\alpha}(\eta, t) \leq \alpha(\eta, t)=\eta(t)$, we only have to check that $\widetilde{\alpha}(\eta, t) \geq \eta(t)$. Since the claim is trivial if $\eta(t)=0$, we can assume that $t \in[0,(d-1) / d)$. Then, by equation (16), there exists a constant $C_{2}$ and a neighborhood $V$ of $t$ such that, for any $t_{1}, t_{2}$ in $V$,

$$
\left|\lambda\left(t_{1}\right)-\lambda\left(t_{2}\right)\right| \leq C_{2}\left|t_{1}-t_{2}\right|^{\eta\left(t_{1}\right)},
$$

which implies that, for any $\epsilon>0$,

$$
\widetilde{\alpha}(\eta, t) \geq \eta(t+\epsilon) .
$$

Since $\eta(t+\epsilon) \rightarrow \eta(t)$ as $\epsilon \rightarrow 0$, it follows that $\widetilde{\alpha}(\eta, t) \geq \eta(t)$, as required.

Remark 17. Using the characterization of $\mathcal{U}$ we can also give an elementary proof of the result of Urbański [14, Theorem 2] that states that

$$
\text { H.dim } K(t)=\mathrm{H} \cdot \operatorname{dim}(\mathcal{U} \cap[t, 1]) \quad \text { for all } t \in[0,1] .
$$

Proof. Indeed, if we denote by $\mathcal{P} \subset \mathcal{U}$ the set of elements in $\mathcal{U}$ with a purely periodic base- $d$ expansion, it is easy to check that $\eta(\mathcal{P})$ is dense in $[0,1]$. Therefore, since both the function $\eta(t)$ and $\tilde{\eta}(t):=H \cdot \operatorname{dim}(\mathcal{U} \cap[t, 1])$ are decreasing, to prove our claim it is enough to check that the equality $\eta(t)=\widetilde{\eta}(t)$ holds for all $t \in \mathcal{P}$.

Since $\mathcal{U} \cap[t, 1] \subseteq K(t)$ for any $t$ (see equation (6)), the inequality $\eta(t) \geq \widetilde{\eta}(t)$ is straightforward, so we only have to prove $\eta(t) \leq \widetilde{\eta}(t)$. If $t_{0} \in \mathcal{P}$, then $t_{0}=. \overline{\epsilon_{1} \ldots \epsilon_{m}}$ is a fixed point of the affine contraction associated with the Lyndon word $W:=\epsilon_{1} \ldots \epsilon_{m}$ (see equation (5)). Given any $t_{1}>t_{0}$, we can fix $\ell \geq 1$ such that $W^{\ell} \cdot 1<t_{1}$, and define the set

$$
S:=W^{\ell} \cdot K\left(t_{1}\right) .
$$

It is easy to check that $S \subset \mathcal{U} \cap\left[t_{0}, 1\right]$ so H.dim $S \leq \mathrm{H} . \operatorname{dim}\left(\mathcal{U} \cap\left[t_{0}, 1\right]\right)$. Moreover, since $S$ is an affine copy of $K\left(t_{1}\right), H . \operatorname{dim} S=\mathrm{H} \cdot \operatorname{dim} K\left(t_{1}\right)$.

Thus we have proved that $\widetilde{\eta}\left(t_{0}\right) \geq \eta\left(t_{1}\right)$ for all $t_{1}>t_{0}$, so our claim follows by taking the limit for $t_{1} \rightarrow t_{0}$ and using the continuity of $\eta(t)$.

Acknowledgements. The authors would like to acknowledge the support of the CRM 'Ennio de Giorgi' of Pisa, and thank the anonymous referee for helpful comments. C.C. is partially supported by the GNAMPA group of the 'Istituto Nazionale di Alta Matematica' (INdAM) and the MIUR project 'Teorie geometriche e analitiche dei sistemi Hamiltoniani in dimensioni finite e infinite' (PRIN 2010JJ4KPA_008). He would also like to thank Sabrina Mantaci for some useful discussions on Lyndon words.

\section{REFERENCES}

[1] O. F. Bandtlow and H. H. Rugh. Entropy-continuity for interval maps with holes, Preprint, 2015, arXiv: 1510.06043

[2] W. de Melo and S. van Strien. One-dimensional dynamics. Ergebnisse der Mathematik und ihrer Grenzgebiete (3). Springer, Berlin, 1993, p. 25. 
[3] C. Dettmann. Open circle maps: small hole asymptotics. Nonlinearity 26(1) (2013), 307-317.

[4] J. Guckenheimer. The growth of topological entropy for one-dimensional maps. Global theory of dynamical systems (Proc. Internat. Conf., Northwestern Univertsity, Evanston, IL., 1979) (Lecture Notes in Mathematics, 819). Springer, Berlin, 1980, pp. 216-223.

[5] J. E. Hutchinson. Fractals and self-similarity. Indiana Univ. Math. J. 30(5) (1981), 713-747.

[6] S. Isola and A. Politi. Universal encoding for unimodal maps. J. Stat. Phys. 61(1-2) (1990), 263-291.

[7] G. Keller and C. Liverani. Rare events, escape rates and quasistationarity: some exact formulae. J. Stat. Phys. 135(3) (2009), 519-534.

[8] M. Lothaire. Combinatorics on words. Encyclopedia of Mathematics and its Applications. Addison-Wesley, Reading, MA, 1983, 17.

[9] J. Milnor and W. Thurston. On iterated maps of the interval. Dynamical systems (College Park, MD, 1986-1987) (Lecture Notes in Mathematics, 1342). Springer, Berlin, 1988, pp. 465-563.

[10] M. Moran. Hausdorff measure of infinitely generated self-similar sets. Monatsh. Math. 122(4) (1996), 387-399.

[11] J. Nilsson. On numbers badly approximable by dyadic rationals. Israel J. Math. 171 (2009), 93-110.

[12] G. Tiozzo. Topological entropy of quadratic polynomials and dimension of sections of the Mandelbrot set. Adv. Math. 273 (2015), 651-715.

[13] G. Tiozzo. Continuity of core entropy of quadratic polynomials. Invent. Math. (2015), to appear. doi: 10.1007/s00222-015-0605-9.

[14] M. Urbański. On Hausdorff dimension of invariant sets for expanding maps of a circle. Ergod. Th. \& Dynam. Sys. 6(2) (1986), 295-309.

[15] L. S. Young. Dimension, entropy and Lyapunov exponents. Ergod. Th. \& Dynam. Sys. 2(1) (1982), 109-124. 